\title{
Evidence of Social and Structural COVID-19 Disparities by Sexual Orientation, Gender Identity, and Race/Ethnicity in an Urban Environment
}

\author{
Megan M Ruprecht $\mathbb{D} \cdot$ Xinzi Wang • Amy K Johnson • Jiayi Xu • Dylan Felt • Siobhan \\ Ihenacho • Patrick Stonehouse • Caleb W. Curry • Catherine DeBroux • Diogo Costa • \\ Gregory Phillips II
}

Accepted: 1 November 2020 / Published online: 1 December 2020

(C) The New York Academy of Medicine 2020

\begin{abstract}
The ongoing COVID-19 pandemic has had widespread social, psychological, and economic impacts. However, these impacts are not distributed equally: already marginalized populations, specifically racial/ ethnic minority groups and sexual and gender minority populations, may be more likely to suffer the effects of COVID-19. The COVID-19 Resiliency Survey was conducted by the city of Chicago to assess the impact of COVID-19 on city residents in the wake of Chicago's initial lockdown, with particular focus on the experiences of minority populations. Chi-square tests of independence were performed to compare COVID-19related outcomes and impacts on heterosexual vs. sexual minority populations, cisgender vs. gender minority populations, and White vs. racial/ethnic minority subgroups. Marginalized populations experienced significant disparities in COVID-19 exposure, susceptibility, and treatment access, as well as in psychosocial effects of the pandemic. Notably, Black and Latinx populations reported significant difficulties accessing food and supplies $(p=0.002)$. Healthcare access disparities were also visible, with Black and Latinx respondents reporting significantly lower levels of access to a provider to see if COVID-19 testing would be appropriate $(p=0.013)$, medical services $(p=0.001)$, and use of telehealth for mental health services $(p=0.001)$. Sexual minority
\end{abstract}

M. M. Ruprecht $(\bowtie) \cdot$ X. Wang · A. K. Johnson · J. Xu •

D. Felt $\cdot$ S. Ihenacho $\cdot$ P. Stonehouse $\cdot$ C. W. Curry $\cdot$

C. DeBroux $\cdot$ D. Costa $\cdot$ G. Phillips II

Department of Medical Social Sciences, Northwestern University

Feinberg School of Medicine, Chicago, IL, USA

e-mail: megan.ruprecht@ northwestern.edu respondents reported significantly lower rates of using telehealth for mental health services $(p=0.011)$, and gender minority respondents reported significantly lower levels of primary care provider access $(p=0.016)$. There are evident COVID-19 disparities experienced in Chicago especially for Black, Latinx, sexual minority, and gender minority groups. A greater focus must be paid to health equity, including providing increased resources and supplies for affected groups, adapting to inequities in the built environment, and ensuring adequate access to healthcare services to ameliorate the burden of COVID-19 on these marginalized populations.

Keywords COVID-19 $\cdot$ Health disparities $\cdot$ Sexual and gender minority populations $\cdot$ Racial/ethnic minority populations $\cdot$ Pandemic response

\section{Introduction}

The ongoing pandemic of the novel coronavirus SARSCoV-2 (i.e., COVID-19) has had grave social, psychological, and economic impacts on a global and national scale. As of August 9th, 2020, there have been over 161,000 deaths in the USA alone [15]. Beyond morbidity and mortality, precautionary health measures related to COVID-19 have had devastating economic impacts in the USA, resulting in high rates of unemployment and closed businesses [40]. The medical system has been overwhelmed [37], and social isolation, fear, and grief may be leading to unprecedented levels of COVID-19- 
related traumatic stress and other mental health consequences $[25,26]$.

Historically, infectious diseases have not had an equal impact across society [10]. Therefore, unsurprisingly, the impacts of COVID-19 have been and will likely continue to be felt most by those populations already marginalized by systemic discrimination and oppression [58]. Current national data show that Black, Latinx, and Native American populations are much more likely to contract and suffer from COVID-19 than their White counterparts [16]. While data on COVID-19 among sexual and gender minority (SGM) populations is not widely available due to lack of measurement in electronic health records or public health surveillance [52], available evidence indicates that SGM populations may also be disproportionately burdened by COVID-19 impacts and infection compared to their cisgender, heterosexual peers. Due to existing financial inequality [29], Black and Latinx populations have been disproportionately impacted by the social and financial impacts of COVID-19 [32]. Similarly, as data have already begun to show, SGM populations have also been severely impacted by the social and economic impacts of COVID-19 [27]. As explored by Blumenshine et al., racial/ethnic and other socioeconomic disparities in infectious diseases operate on three levels-exposure, susceptibility, and treatment access - that when combined lead to overall worse health outcomes [10].

Firstly, Black and Latinx people are more likely to be exposed to COVID-19 as they are more likely to have jobs in underpaid "essential industries," which require in-person or face-to-face contact, thereby increasing potential exposure [56], as are many SGM populations [27]. Institutional discrimination within built environment, specifically high housing density as a result of racist zoning laws, also makes physical distancing more challenging in many communities of color [3, 60]. Moreover, as a result of racist policing practices and systemic injustices within the court system, Black and Latinx individuals are highly overrepresented in the prison population [41], as are SGM populations, particularly Black and Latinx SGM [7]. This is a critical element of exposure given that COVID-19 has been shown to spread rapidly in carceral spaces [24].

There are furthermore visible disparities in susceptibility for marginalized populations. Underlying conditions associated with COVID-19 morbidity and mortality, such as asthma [18, 23], diabetes [8, 49, 59], unsuppressed HIV viral load [17, 42, 59], and cardiovascular diseases [18, 45, 48], are more prevalent in Black and Latinx populations, as well as in SGM. This places these groups at higher risk for severe cases of COVID-19. SGM individuals may have greater susceptibility to COVID-19, due to their more frequent use of cigarettes and marijuana (known risk factors for COVID-19 morbidity) than their cisgender, heterosexual counterparts [53]. Finally, existing disparities in rates of psychological stress and trauma may impact the susceptibility of certain groups to COVID-19-related trauma and mental distress [5]. For example, higher rates of mental health problems in SGM populations as a result of discrimination and minority stress and lower levels of social support may make coping with the psychological challenges of isolation and trauma associated with the COVID-19 pandemic more difficult [11, 22]. The higher rates of certain mental health conditions including trauma in Black and Latinx populations place them similarly at risk for psychological conditions to be worsened by the pandemic $[38,46]$.

Finally, in regard to treatment access, COVID-19 has amplified existing healthcare disparities in highly visible ways with issues such as lack of healthcare facilities and lack of health insurance becoming even more pertinent for racial/ethnic minority and SGM populations [35, 55]. Not only may Black and Latinx individuals be less likely to seek care due to cost, lack of insurance, medical mistrust, and a lack of appropriate healthcare facilities in one's own community [6] but also may receive substandard care after treatment is sought due to racism at pointof-care [39]. This is compounded by other structural and neighborhood-level issues including problems accessing childcare, transportation, and linguistic barriers $[14,36]$. SGM populations are also highly likely to experience gaps in receipt of timely and effective treatment. Due to both financial barriers to care, lower rates of insurance, medical mistrust, and discrimination at point-of-care, SGM individuals report lower rates of healthcare engagement [9, 34]. These effects are further magnified for multiply marginalized individuals, including racial/ethnic minority SGM groups [34]. Furthermore, expanding beyond just disparities in physical impacts, both racial/ethnic minority and SGM populations report even greater disparities in accessing quality, culturally competent mental healthcare $[1,51]$, meaning that they may not be able to get appropriate professional support for the psychosocial distress brought on by the pandemic. 
These disparities in exposure, susceptibility, and access did not arise spontaneously during the COVID-19 pandemic [13]. Racism, White supremacy, homo/biphobia, transphobia, and other forms of structural violence have constructed social conditions which operate at all three disparity levels, impacting Black, Latinx, and SGM peoples' likelihood of being exposed to the virus, risk of contracting the disease when exposed, and likelihood of receiving timely and effective treatment after infection. These structures thereby increase exposure and susceptibility, while reducing access to resources for marginalized populations [30], including racial, ethnic, sexual, and gender minority individuals [28]. Further investigation into the specific social and material support needs of these communities is critical in order to ameliorate the ongoing damages of COVID-19 in marginalized populations.

\section{The Current Study}

The city of Chicago, like most US cities, has seen drastic disparities in COVID-19 incidence and mortality. Roughly $68 \%$ of COVID-19-related deaths and 52\% of COVID-19 cases have been among Black residents [61], though only $30 \%$ of the city's population is Black [57]. Similarly, while just $29 \%$ of Chicago's total population identifies as Latinx [57], this group represents $31.5 \%$ of COVID-19-related deaths and $47.7 \%$ of cases [61]. Such disparities, while troubling, are not surprising given the widespread racial segregation and discrimination in Chicago [43]. Further data is needed to inform strategies for equitable and effective future pandemic control and response particularly in urban areas.

\section{Methods}

As part of a Racial Equity Rapid Response (RERR) initiative, the Mayor's office conducted a COVID-19 Resiliency Survey to assess the impacts of COVID-19 on city residents at the start of Chicago's initial lockdown, with particular focus on the experiences of minority populations. The survey included questions about COVID-19 symptoms, testing, vulnerabilities, behavioral patterns, structural barriers to health, and social needs. Notably, the Chicago COVID-19 Resiliency Survey was one of few surveys nationally about COVID-19 to include questions about sexual orientation and gender identity. The survey was designed to identify unmet needs among marginalized groups in order to tailor social services and inform a more equitable COVID19 response.

The survey sample consisted of 227 participants, ranging in age from 21 to over 70 years. Only participants indicating valid ZIP codes within Chicago were included in analyses, resulting in a final sample of 206 participants. Data were collected between 4/16/2020 and 5/14/2020 during phases I and II of the pandemic. This unpaid survey was distributed online and disseminated via Listservs and community agencies, with a focus on reaching citizens on the south and west sides who were most affected by the pandemic.

\section{Survey Design}

\section{Measures}

\section{Demographics}

Sexual Identity Sexual identity was assessed by asking, "Which of the following terms do you use to describe your sexual orientation?" Response options included gay, lesbian, bisexual, pansexual, queer, heterosexual, some other terms, and prefer not to respond. Due to sample size limitations, all sexual minority subgroups (gay, bisexual, lesbian, and queer) were collapsed to form a "sexual minority" category; this is a non-ideal method for analysis which should only be considered appropriate in cases where analysis would not be possible with subgroup differentiation.

Gender Gender modality - that is, the state of being transgender or cisgender [4] — was assessed by asking participants if they identified as transgender (yes or no). Gender identity was then assessed by asking participants to select the terms they use to describe their gender from the following list: woman, man, non-binary, some other term, and prefer not to respond. Due to sample size limitations, participants were categorized and were collapsed to form one "gender minority" category if they either selected "yes" in the gender modality question or selected "non-binary" in the gender identity question. All other responses were categorized as cisgender. We refer to this construct as gender modality moving forward, as we are not distinguishing participants based on their gender alone (i.e., male or female) but rather by their relation to their assigned sex at birth. 
Race/Ethnicity Race and ethnicity were assessed by combining two questions. First, participants were asked if they identified as Hispanic or Latino/a/x (yes or no). If participants endorsed a Hispanic/Latinx identity, their race/ethnicity was coded as such, regardless of reported race. If they did not, their race was categorized in accordance with their response to the question "How do you describe your race? Please chose all that apply to you?" with the response options being American Indian or Alaska Native, Asian, Black or African American, Native Hawaiian or other Pacific Islander, White, and not listed. Hence, the response categories were (1) Black or African American, (2) Hispanic/Latinx (regardless of reported race), (3) White, and (4) other.

\section{COVID-19 Exposure}

Exposure was assessed by asking about symptoms, household members having symptoms, feelings of safety at home and commuting to work, and prevention behaviors, including physical distancing, masks, isolation, and washing hands (See Supplementary Table 1 for further detail).

\section{COVID-19 Susceptibility}

Susceptibility to the negative impacts of COVID-19, both physically and mentally, was assessed by asking about chronic compromise of immune system among household members, shortages of food and sanitation supplies, support from community organizations and mutual aid services, spending time with household, feeling alone/isolated, and access to technology (See Supplementary Table 1 for further detail).

\section{COVID-19 Treatment Access}

Access to treatment, both for COVID-19 and for mental health distress related to the pandemic, was assessed by asking about access to medical services, health insurance, a primary care provider, a mental healthcare provider, and a medical provider to indicate whether or not COVID-19 testing is appropriate. Usage of telehealth for mental health services, telehealth for physical health services, and apps for mental health support was also assessed (See Supplementary Table 1 for further detail).

\section{Statistical Analysis}

All data cleaning, recoding, and analyses were conducted in SAS version 9.4 (SAS Institute, Cary, NC). Descriptive analyses were conducted to determine the prevalence of sexual identity, gender modality, race/ethnicity, and other demographics within the entire sample. Chi-square tests of independence were performed to compare COVID-19-related outcomes in heterosexual vs. sexual minority populations, in cisgender vs. gender minority populations, and between racial/ ethnic subgroups; statistically significant $(p<0.05)$ results are reported below.

\section{Results}

\section{Demographics}

The sample was racially/ethnically diverse; $51.9 \%(n=$ $107)$ of the sample was Black/African American, $31.1 \%$ $(n=64)$ White, $12.1 \%(n=25)$ Hispanic/Latinx, and less than $2 \%$ of each of the remaining identities (Table 1). Similarly, a diverse range of sexual identities was represented in the sample: $62.6 \%$ of respondents identified as straight/heterosexual $(n=129), 13.1 \%$ as gay $(n=27)$, $6.3 \%$ as queer $(n=13), 6.3 \%$ as bisexual $(n=13), 2.9 \%$ as lesbian $(n=6)$, and $5.8 \%$ preferred not to respond $(n=$ 12). A small portion of the sample endorsed a gender minority identity: $1.9 \%$ of the sample $(n=4)$ reported that they were transgender, and $3.4 \%$ of the sample reported that they were non-binary $(n=7)$, not mutually exclusive. There was a broad representation of ages, from 21 to over 70 years, with $38.8 \%$ of the respondents under $40(n=87)$, $40.6 \%$ between 40 and $59(n=91)$, and $19.2 \%$ of the sample 60 or older $(n=43)$. The sample was geographically spread throughout Chicago, with the north and south sides especially represented (Fig. 1).

\section{Disparities in Exposure}

Despite high overall levels of adherence, White respondents were significantly more likely to report adherence to physical distancing than Black and Latinx participants (W: $100.0 \%$ vs. B: $90.4 \%$ vs. L: $92.0 \% ; \chi^{2}=$ $6.24 ; p=0.044)$. White respondents were also significantly more likely to report adherence to quarantine than Black or Latinx participants (W: $82.3 \%$ vs. B: $58.7 \%$ vs. L: $\left.76.0 \% ; \chi^{2}=10.78 ; p=0.005\right)$. There were no 
Table 1 Characteristics of survey respondents

\begin{tabular}{|c|c|c|}
\hline & \multicolumn{2}{|l|}{ Total } \\
\hline & $N$ & $\%$ \\
\hline \multicolumn{3}{|l|}{ Race/ethnicity } \\
\hline Black & 104 & 54.5 \\
\hline Hispanic & 25 & 13.1 \\
\hline White & 62 & 32.5 \\
\hline American Indian or Alaska Native & 3 & 1.3 \\
\hline Native Hawaiian or other Pacific Islander & 2 & 0.9 \\
\hline Asian & 5 & 2.2 \\
\hline \multicolumn{3}{|l|}{ Sexual identity } \\
\hline Heterosexual & 129 & 64.5 \\
\hline Gay & 27 & 13.1 \\
\hline Bisexual & 13 & 6.3 \\
\hline Lesbian & 6 & 2.9 \\
\hline Queer & 13 & 6.3 \\
\hline Prefer not to respond & 12 & 5.8 \\
\hline \multicolumn{3}{|l|}{ Gender modality } \\
\hline Cisgender & 196 & 95.2 \\
\hline Transgender and/or non-binary & 10 & 4.8 \\
\hline \multicolumn{3}{|l|}{ Age, years } \\
\hline $21-29$ & 28 & 13.7 \\
\hline $30-39$ & 55 & 27.0 \\
\hline $40-49$ & 39 & 19.1 \\
\hline 50-59 & 45 & 22.1 \\
\hline $60-69$ & 33 & 16.2 \\
\hline $70+$ & 3 & 1.5 \\
\hline
\end{tabular}

statistically significant differences in any other preventive behaviors, such as mask wearing or washing hands (Table 2), which had high reported adherence across racial/ethnic groups. Latinx respondents were also more than twice as likely to report feeling unsafe going to work and/or commuting to work compared to White and Black participants (L: $48.0 \%$ vs. W: $19.3 \%$ vs. B: $19.2 \% ; \chi^{2}=10.11 ; p=0.006$ ) (Table 2). There were no statistically significant differences in exposure, including testing or preventive behaviors, by sexual identity or gender modality (Tables 3 and 4).

\section{Disparities in Susceptibility}

White respondents were more likely to report having no household members with compromised immune systems compared with Black and Latinx respondents
(W: $61.3 \%$ vs. B: $36.5 \%$ vs. L: $40.0 \% ; \chi^{2}=9.91 ; p=$ 0.007). While $17.3 \%$ of Black and $8.0 \%$ of Latinx participants reported experiencing food shortages during the pandemic, not a single White respondent reported this experience $\left(\chi^{2}=12.60 ; p=0.002\right)$. Similarly, Black and Latinx participants frequently reported shortages in sanitation or cleaning supplies though White respondents were significantly less likely to report these same shortages (B: $45.2 \%$ vs. L: $40.0 \%$ vs. W: $17.7 \%$; $\chi^{2}=13.01 ; p=0.002$; Table 2). There were no significant differences in household members with compromised immune systems or reported food/supply shortages by sexual identity or gender modality.

Participants impacted by systemic discrimination also reported factors that suggest higher susceptibility to mental health problems during the pandemic. For example, there were statistically significant differences in the amount of social support received: while Latinx and White participants reported similar rates of feeling alone/isolated, Black respondents noted significantly lower levels of feeling alone/isolated (W: $50.0 \%$ vs. L: $52.0 \%$ vs. B: $\left.32.7 \% ; \chi^{2}=6.30 ; p=0.043\right)$. Heterosexual participants reported overall greater levels of social support: they were less likely to feel alone/isolated compared to sexual minority participants $(34.1 \%$ vs. 53.5\%; $\chi^{2}=7.13 ; p=0.008$; Table 3). Heterosexual participants were additionally more likely to spend time with household members compared to sexual minority respondents ( $54.3 \%$ vs. $\left.38.0 \% ; \chi^{2}=4.83 ; p=0.028\right)$. Gender minority respondents were over twice as likely to report feeling alone/isolated compared to cisgender respondents ( $90.0 \%$ vs. $40.3 \% ; \chi^{2}=9.60 ; p=0.002$ ) (Table 4). No other significant differences in social support or connectedness were found.

Respondents differed by race/ethnicity in terms of how they connected with others: Black and Latinx respondents were more likely to access support from a community organization in their area $(17.3 \%$ and $16.0 \%$ of participants), compared to only $1.6 \%$ of White participants $\left(\chi^{2}=\right.$ 9.46; $p=0.009$ ). White participants were, however, more likely to report accessing videoconferencing tools (such as Zoom, Skype, Google Hangouts, Facetime; 87.1\%) compared to $69.2 \%$ of Black respondents and $72.0 \%$ of Latinx respondents $\left(\chi^{2}=6.96 ; p=0.032\right)$.

\section{Disparities in Treatment}

Overall, White respondents had greater access to physical and mental health medical services: they were 


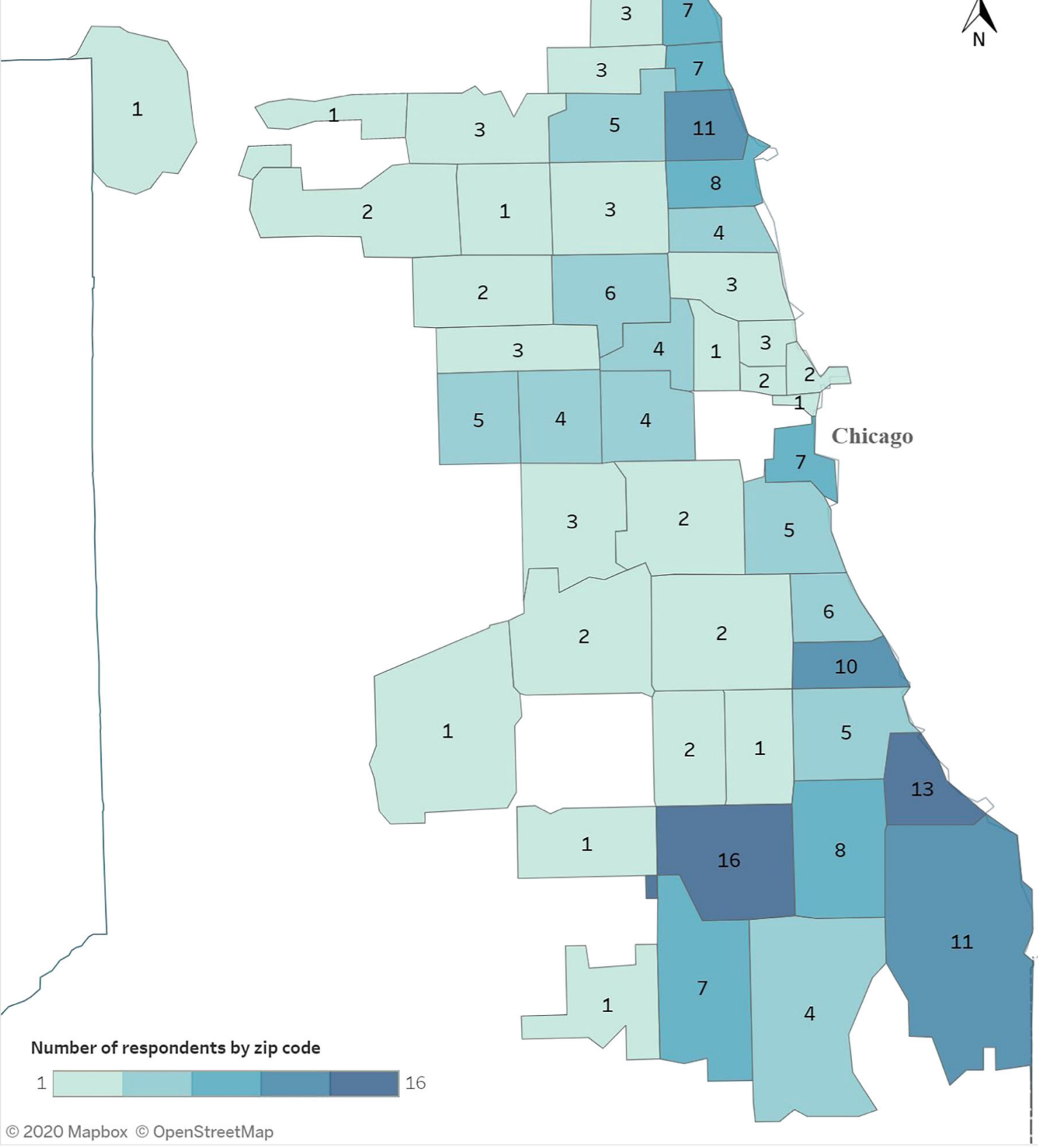

Fig. 1 Geographic distribution of survey participants

significantly more likely to report accessing telehealth from a mental health professional (W: $30.7 \%$ vs. B: 9.7\% vs. L: $\left.16.0 \% ; \chi^{2}=13.48 ; p=0.001\right)$ and more likely to have a medical provider to contact if they felt that they needed COVID-19 testing (W: $96.8 \%$ vs. B: $89.4 \%$ vs. L: $76.0 \% ; \chi^{2}=8.68 ; p=0.013$ ). Black and Latinx respondents were more likely to report barriers to accessing medical services, such as emergency care, basic medical care, or needed prescriptions compared to White participants (B: $53.9 \%$ vs. L: $32.0 \%$ vs. W: 25.8\%; $\chi^{2}=13.70 ; p=0.001$, Table 2). Heterosexual participants had greater access to mental health treatment in particular compared to sexual minority participants, as they were more than twice as likely to report 
Table 2 Differences in COVID-19 social needs by race/ethnicity

\begin{tabular}{|c|c|c|c|c|c|}
\hline & $\begin{array}{l}N=62 \\
\text { White } N(\%)\end{array}$ & $\begin{array}{l}N=104 \\
\text { Black } N(\%)\end{array}$ & $\begin{array}{l}N=25 \\
\text { Latinx } N(\%)\end{array}$ & $\chi^{2}$ & $p$ value \\
\hline \multicolumn{6}{|l|}{ Exposure } \\
\hline Experienced fever, dry couth, and/or shortness of breath & $7(11.3)$ & $13(12.5)$ & $3(12.0)$ & 0.64 & 0.958 \\
\hline Have household members with COVID(-like) symptoms & $2(3.2)$ & $7(6.7)$ & $2(8.0)$ & 1.15 & 0.564 \\
\hline Feeling unsafe at home & $4(6.5)$ & $12(11.5)$ & $6(24.0)$ & 5.38 & 0.068 \\
\hline Feeling unsafe commuting to work & $12(19.3)$ & $20(19.2)$ & $12(48.0)$ & 10.11 & 0.006 \\
\hline \multicolumn{6}{|l|}{ Prevention behaviors } \\
\hline Physical distancing & $62(100.0)$ & $94(90.4)$ & $23(92.0)$ & 6.24 & 0.044 \\
\hline Masks & $58(93.6)$ & $92(88.5)$ & $23(92.0)$ & 1.25 & 0.536 \\
\hline Isolation & $51(82.3)$ & $61(58.6)$ & $19(76.0)$ & 10.78 & 0.005 \\
\hline Washing hands & $62(100.0)$ & $96(92.3)$ & $22(88.0)$ & 6.30 & 0.043 \\
\hline \multicolumn{6}{|l|}{ Susceptibility } \\
\hline No household members w/ chronic compromise of immune system & $38(61.3)$ & $38(36.5)$ & $10(40.0)$ & 9.91 & 0.007 \\
\hline Shortage of food & $0(0.0)$ & $18(17.3)$ & $2(8.0)$ & 12.60 & 0.002 \\
\hline Shortage of sanitation/cleaning supplies & $11(17.7)$ & $47(45.2)$ & $10(40.0)$ & 13.01 & 0.002 \\
\hline Support from community organization & $1(1.6)$ & $18(17.3)$ & $4(16.0)$ & 9.46 & 0.009 \\
\hline Mutual aid services & $2(3.2)$ & $10(9.6)$ & $1(4.0)$ & 2.86 & 0.240 \\
\hline Spending time with household & $36(58.1)$ & $41(39.4)$ & $15(60.0)$ & 7.02 & 0.030 \\
\hline Feeling alone/isolated & $31(50.0)$ & $34(32.7)$ & $13(52.0)$ & 6.30 & 0.043 \\
\hline Lack of technology & $1(1.6)$ & $9(8.6)$ & $3(12.0)$ & 4.26 & 0.118 \\
\hline \multicolumn{6}{|l|}{ Treatment access } \\
\hline Access to medical services & $16(25.8)$ & $56(53.8)$ & $8(32.0)$ & 13.70 & 0.001 \\
\hline Access to health insurance/care coverage & $59(95.2)$ & $94(90.4)$ & $21(84.0)$ & 2.99 & 0.237 \\
\hline Access to primary care provider/physician & $57(91.9)$ & $91(87.5)$ & $19(76.0)$ & 4.12 & 0.128 \\
\hline Access to mental health provider & $30(48.4)$ & $39(37.5)$ & $7(28.0)$ & 3.59 & 0.166 \\
\hline Access to medical provider to indicate appropriateness of testing & $60(96.8)$ & $93(89.4)$ & $19(76.0)$ & 8.68 & 0.013 \\
\hline Mental telehealth & $19(30.7)$ & $9(8.7)$ & $4(16.0)$ & 13.48 & 0.001 \\
\hline Physician telehealth & $14(22.6)$ & $19(18.3)$ & $5(20.0)$ & 0.45 & 0.797 \\
\hline Use of apps for mental health support & $10(16.1)$ & $10(9.6)$ & $6(24.0)$ & 4.04 & 0.133 \\
\hline
\end{tabular}

accessing telehealth from a mental health professional (26.8\% vs. $\left.12.4 \% ; \chi^{2}=6.54 ; p=0.011\right)$, but were less likely to have a mental health provider $(34.9 \%$ vs. $\left.52.1 \% ; \chi^{2}=5.62 ; p=0.018\right)$ compared to sexual minority participants (Table 3 ). Gender minority respondents experienced significant disparities in access to physical, though not mental, healthcare: cisgender participants were significantly more likely to have a primary care provider or physician $\left(87.2 \%\right.$ vs. $60.0 \% ; \chi^{2}=$ $5.84 ; p=0.016$ ) compared to gender minority respondents. Cisgender respondents were, however, less likely to report accessing telehealth from a mental health professional compared to gender minority respondents ( $14.8 \%$ vs. $60.0 \% ; \chi^{2}=13.79 ; p=0.001$; Table 4 ).

\section{Discussion}

As we would predict based on the work of Blumenshine et al., there were significant disparities in COVID-19 exposure, susceptibility, and treatment access, both for the virus itself and related psychosocial consequences, among marginalized populations. These structural determinants are, however, unsurprising given the omnipresent and pervasive stigma and discrimination that impact racial/ethnic, sexual, and gender minority populations in Chicago.

While there were significant differences by race/ ethnicity in preventive behaviors, it is critical to examine these from a structural, rather than individual, level 
Table 3 Differences in COVID-19 social needs by sexual identity

\begin{tabular}{|c|c|c|c|c|}
\hline & $\begin{array}{l}N=129 \\
\text { Heterosexual } N(\%)\end{array}$ & $\begin{array}{l}N=71 \\
\text { Sexual minority } N(\%)\end{array}$ & $\chi^{2}$ & $p$ value \\
\hline \multicolumn{5}{|l|}{ Exposure } \\
\hline Experienced fever, dry couth, and/or shortness of breath & $21(16.3)$ & $6(8.5)$ & 2.61 & 0.272 \\
\hline Have household members with COVID(-like) symptoms & $6(8.5)$ & $2(2.8)$ & 2.46 & 0.117 \\
\hline Feeling unsafe at home & $16(12.4)$ & $8(11.3)$ & 0.06 & 0.813 \\
\hline Feeling unsafe commuting to work & $26(20.2)$ & $19(26.8)$ & 1.15 & 0.284 \\
\hline \multicolumn{5}{|l|}{ Prevention behaviors } \\
\hline Physical distancing & $121(93.8)$ & $64(90.1)$ & 0.88 & 0.347 \\
\hline Masks & $117(90.7)$ & $63(88.7)$ & 0.20 & 0.658 \\
\hline Isolation & $86(66.7)$ & $51(71.8)$ & 0.05 & 0.827 \\
\hline Washing hands & $123(95.4)$ & $66(93.0)$ & 0.57 & 0.452 \\
\hline \multicolumn{5}{|l|}{ Susceptibility } \\
\hline No household members w/ chronic compromise of immune system & $63(48.8)$ & $30(42.4)$ & 0.80 & 0.372 \\
\hline Shortage of food & $15(11.6)$ & $7(9.9)$ & 0.15 & 0.702 \\
\hline Shortage of sanitation/cleaning supplies & $45(34.9)$ & $24(33.8)$ & 0.02 & 0.879 \\
\hline Support from community organization & $17(13.2)$ & $6(8.4)$ & 1.00 & 0.316 \\
\hline Mutual aid services & $8(6.2)$ & $5(7.0)$ & 0.05 & 0.818 \\
\hline Spending time with household & $70(54.3)$ & $27(38.0)$ & 4.83 & 0.028 \\
\hline Feeling alone/isolated & $44(34.1)$ & $38(53.5)$ & 7.13 & 0.008 \\
\hline Lack of technology & $8(6.2)$ & $4(5.6)$ & 0.03 & 0.872 \\
\hline \multicolumn{5}{|l|}{ Treatment access } \\
\hline Access to medical services & $49(38.0)$ & $33(46.5)$ & 1.37 & 0.243 \\
\hline Access to health insurance/care coverage & $119(92.3)$ & $63(88.7)$ & 0.69 & 0.406 \\
\hline Access to primary care provider/physician & $112(86.8)$ & $62(87.3)$ & 0.01 & 0.919 \\
\hline Access to mental health provider & $45(34.9)$ & $37(52.1)$ & 5.62 & 0.018 \\
\hline Access to medical provider to indicate appropriateness of testing & $118(91.5)$ & $63(88.7)$ & 0.40 & 0.527 \\
\hline Mental telehealth & $16(12.4)$ & $19(26.8)$ & 6.54 & 0.011 \\
\hline Physician telehealth & $22(17.1)$ & $17(23.9)$ & 1.38 & 0.239 \\
\hline Use of apps for mental health support & $13(10.1)$ & $14(19.7)$ & 3.65 & 0.056 \\
\hline
\end{tabular}

perspective to inform effective intervention strategy, since these are likely attributable to larger economic and social disparities. For example, Black and Latinx individuals reported less physical distancing and quarantine compared to White respondents. This difference is likely a result of systemic factors related to the built environment, which made physical distancing and isolation more difficult for many within these populations, not individual choice. These include high-density housing, larger household/ family size, working in "essential services," and being unable to take time off work due to economic insecurity and a lack of paid sick time [30]. The reported shortage of cleaning and sanitation supplies also likely contribute to differences in preventive behavior. Thus, to increase preventive behaviors, it is not enough to provide education or inform about the proper precautions. Rather, policylevel changes, including guaranteed paid sick leave, an expansion of safe and affordable housing, universal basic income, expansion of unemployment benefits during the pandemic, and increased availability of resources, must be undertaken to ensure that adherence to preventive measures are equitable across subpopulations. In the short term, however, increased guidance, drafted in collaboration with community leaders, should be made for how to physical distance for families in high-density housing or facing other structural obstacles to engaging in preventive behavior. Safety remains a concern for some groups: a staggering $48 \%$ of Latinx participants reported feeling 
Table 4 Differences in COVID-19 social needs by gender modality

\begin{tabular}{|c|c|c|c|c|}
\hline & $\begin{array}{l}N=196 \\
\text { Cisgender } N(\%)\end{array}$ & $\begin{array}{l}N=10 \\
\text { Gender minority } N(\%)\end{array}$ & $\chi^{2}$ & $p$ value \\
\hline \multicolumn{5}{|l|}{ Exposure } \\
\hline Experienced fever, dry couth, and/or shortness of breath & $25(12.2)$ & $3(30.0)$ & 3.58 & 0.167 \\
\hline Have household members with COVID(-like) symptoms & $12(6.1)$ & $1(10.0)$ & 0.24 & 0.623 \\
\hline Feeling unsafe at home & $23(11.7)$ & $2(20.0)$ & 0.61 & 0.435 \\
\hline Feeling unsafe commuting to work & $43(21.9)$ & $5(50.0)$ & 4.19 & 0.041 \\
\hline \multicolumn{5}{|l|}{ Prevention behaviors } \\
\hline Physical distancing & $181(92.4)$ & $10(100.0)$ & 0.83 & 0.364 \\
\hline Masks & $176(89.8)$ & $10(100.0)$ & 1.13 & 0.288 \\
\hline Isolation & $133(67.9)$ & $8(80.0)$ & 0.65 & 0.420 \\
\hline Washing hands & $184(93.9)$ & $10(100.0)$ & 0.65 & 0.420 \\
\hline \multicolumn{5}{|l|}{ Susceptibility } \\
\hline No household members w/ chronic compromise of immune system & $92(46.9)$ & $4(40.0)$ & 0.18 & 0.668 \\
\hline Shortage of food & $21(10.7)$ & $1(10.0)$ & $<0.01$ & 0.943 \\
\hline Shortage of sanitation/cleaning supplies & $69(35.2)$ & $2(20.0)$ & 0.97 & 0.324 \\
\hline Support from community organization & $23(11.7)$ & $1(10.0)$ & 0.03 & 0.868 \\
\hline Mutual aid services & $11(5.6)$ & $2(20.0)$ & 2.25 & 0.133 \\
\hline Spending time with household & $94(48.0)$ & $5(50.0)$ & 0.016 & 0.997 \\
\hline Feeling alone/isolated & $79(40.3)$ & $9(90.0)$ & 9.60 & 0.002 \\
\hline Lack of technology & $14(7.1)$ & $0(0.0)$ & 0.77 & 0.381 \\
\hline \multicolumn{5}{|l|}{ Treatment access } \\
\hline Access to medical services & $81(41.3)$ & $4(40.0)$ & $<0.01$ & 0.934 \\
\hline Access to health insurance/care coverage & $177(90.3)$ & $9(90.0)$ & $<0.01$ & 0.976 \\
\hline Access to primary care provider/physician & $171(87.2)$ & $6(60.0)$ & 5.84 & 0.016 \\
\hline Access to mental health provider & $73(37.3)$ & $6(60.0)$ & 1.70 & 0.193 \\
\hline Access to medical provider to indicate appropriateness of testing & $177(90.3)$ & $8(80.0)$ & 1.10 & 0.293 \\
\hline Mental telehealth & $29(14.8)$ & $6(60.0)$ & 13.79 & $<0.001$ \\
\hline Physician telehealth & $37(18.8)$ & $3(30.0)$ & 0.75 & 0.386 \\
\hline Use of apps for mental health support & $25(12.8)$ & $3(30.0)$ & 2.41 & 0.121 \\
\hline
\end{tabular}

unsafe on their way to/from work. This underscores the importance of investing in community infrastructure and providing accessible personal protective equipment (PPE) for all who need it.

Differences in susceptibility similarly underscore the importance of investing in communities. Black and Latinx respondents and their households showed greater susceptibility to COVID-19, being more likely to report having a family member with a compromised immune system. This is in line with previous research which stated that Black and Latinx populations had higher risk for COVID-19 morbidity and mortality [47]. The combination of higher levels of vulnerability and decreased ability to engage in preventive behaviors, along with systemic racism within the medical system, contributes to the higher levels of COVID-19 morbidity and mortality seen among racial/ethnic minority populations $[19,30,55]$. It furthermore creates an added urgency in deploying supplies, food, PPE, financial assistance, and other emergency measures to impacted communities. This can subsequently help ameliorate these negative outcomes, in conjunction with structural change, to combat the higher levels of vulnerability seen in Black and Latinx populations. Black and Latinx respondents also reported significantly greater trouble accessing food and basic supplies. However, it is difficult to know if this is due to store closures, individuals not being able to shop due to risk of exposure, or because of financial 
difficulties caused by COVID-19. Therefore, further investigation into the specific food and supply needs of vulnerable populations is warranted to inform more targeted intervention. Still, immediate relief measures can be taken in the forms of emergency food supply delivery, expansion of unemployment benefits, and provisions of stipends to communities disproportionately impacted by COVID-19.

Clear deficits in social needs appeared for certain populations, which could represent higher susceptibility to psychological distress: SGM respondents reported high levels of feeling alone and isolated. As these two populations already have higher rates of mental health concerns $[11,22]$, finding methods to increase social support while maintaining physical distancing is critical for SGM populations. Still, while online support groups or social events may be a useful tool for creating this support, they may also leave out those who do not have a stable internet connection. Furthermore, Black and Latinx populations reported lower overall use of videoconferencing technologies to maintain social connection. This could reflect differences in the accessibility of technology_Black and Latinx adults are less likely to have access to a laptop and high-speed internet in their home [44]. Therefore, ensuring that all households have access to low-cost, high-speed internet is critical, especially as increasingly more essential functions of everyday life, including schooling, move online. Expanding Wifi access and improving the quality and consistency of Internet, especially in low-income neighborhoods, should remain a priority for local governments. However, these differences also should force caution in the meantime regarding an overreliance on the implementation of online services; an overreliance on internet-based technologies for COVID support may disadvantage populations who struggle to access internet.

One of the most obvious disparities was in access to healthcare. Black, Latinx, and gender minority participants were less likely to report having a primary care provider and were less likely to have a doctor to contact regarding COVID-19 testing. Black and Latinx respondents, furthermore, experienced greater problems in accessing medical services more generally. While differences for sexual minority populations were not statistically significant, they too reported lower levels of healthcare engagement in many areas. This is consistent with previous literature which shows lower rates of healthcare engagement among SGM populations and racial/ethnic minority populations [34, 39]. As such, these disparities in healthcare access and engagement exist outside of the context of COVID-19. Expanding access to affordable, culturally competent, and highquality healthcare located within communities must be a priority to ensure long-term health equity. One area in which SGM populations did have higher access to healthcare was in mental health, which was an unexpected resilience factor. For example, gender minority participants were more likely to have accessed care from a mental health professional, which may be a sign of proactive steps taken to ensure one's mental wellbeing during a pandemic. Of course, this result is difficult to interpret with certainty due to the limited information available. Given the higher overall rates of mental health concerns in SGM populations [11, 31], this specific group of respondents may have simply been more likely to seek mental health treatment for existing conditions. This higher rate of mental healthcare access could also be attributable to gender minority individuals experiencing crisis-level outcomes as a result of severe marginalization, and therefore requiring greater interaction with mental health professionals during the pandemic. Thus, further research is needed to investigate which factors promote mental healthcare utilization within this group.

While lacking in resources and social needs in many areas, the minority populations in our sample also reported resiliency-promoting factors in a number of areas. For example, Black and Latinx participants reported feeling, on average, less alone and more likely to access a community organization in their area than White individuals. These are critical assets which must be taken into account when planning for equity-based pandemic responses. In particular, the increased levels of community organization usage among Black and Latinx respondents suggests that community organizations may be a critical resource to support marginalized populations during times of crisis, as they are able to rapidly fill gaps in social programs and services. Future research should be dedicated to exploring the role of community organizations in supporting equity during health crises, and resources should be made available to support expansion of existing community organization services.

This study is not without limitations. Due to sample size, we collapsed all sexual minority respondents into a single category and all gender minority respondents into a single category. Certain subpopulations, including bisexuals, are known to experience worse health 
outcomes, and as such, further research with these specific groups must be done to disentangle their experience from that of the larger sexual minority community [20]. The specific measures of gender modality used may have underestimated the actual number of gender minority individuals in the sample, for example, if someone did not currently identify as transgender but had a gender identity different than their sex assigned at birth. One of the largest limitations is the omission of Native Americans from more detailed analyses due to small sample size. However, given the large COVID-19 disparities impacting this population in particular, further work, especially focusing on the social needs and structural barriers to health among Indigenous populations in Chicago, is needed. The small sample size also prevents an intersectional analytic approach, meaning that the experiences of multiply marginalized groups cannot be examined statistically with the current sample [12]. For example, while family support may serve as a form of resilience against racial discrimination, Black and Latinx SGM individuals may have diminished access to these social supports due to their sexual/gender minority identity $[2,50]$. Due to racism within the SGM community, racial/ethnic minority SGM populations may be excluded from these community spaces, causing a deficit in another resiliency-promoting factor [21]. Multiply marginalized groups experienced a number of health disparities pre-pandemic: for example, $18.6 \%$ of White transgender individuals lived in poverty in 2019 , compared to $38.5 \%$ of Black transgender people and $48.4 \%$ of Hispanic/Latinx transgender people [54]. As such, not investigating COVID-19-related impacts at these intersections may be obfuscating critical disparities and areas for intervention. Further exploration, including qualitative work and analyses with a larger sample, must be done to elucidate these experiences. All data were self-report; this could have led to an underreporting of nonadherence to COVID-19 prevention measures due to social desirability bias, though the anonymity of the survey helps to reduce the impact of this limitation. Due to sample size limitations, analyses did not control for potential confounders and results should therefore be interpreted with caution. Due to the cross-sectional nature of the study, we cannot infer causality, nor do we know concretely if the patterns we observed among respondents were a direct result of COVID-19 and related shutdown measures, or if these patterns existed before the pandemic. Finally, results of the survey were interpreted using the levels conceived by Blumenshine et al.- exposure, susceptibility, and treatment access; however, given the design of the survey, the metrics do not perfectly align with these levels. Regardless, it remains a useful framework for understanding COVID-19-related disparities.

Despite these limitations, these early results provide essential information which should be used both as COVID-19 persists and in the case of future pandemics which will, undoubtedly, disparately impact marginalized populations. Taking into account the findings from this survey, we have generated four critical recommendations, applicable both to the city of Chicago and any other jurisdictions experiencing similar disparities.

Invest Directly in Communities Black and Latinx respondents reported relatively high utilization of community organizations. As such, funding and supplies for local, community-based organizations should be made readily available so they can respond most effectively to those they serve, particularly given their demonstrated role in supporting communities of color as captured in our results. Giving communities themselves direct ownership over supplies and resources promotes equitable distribution and greater access for marginalized populations.

Collect and Report Comprehensive Data Which Includes Measures of Race/Ethnicity, Sex, Sexual Orientation, and Gender Identity/Modality The exact magnitude of disparities in COVID-19 morbidity and mortality among SGM populations is unknown due to the lack of consistent data collection. Critically, intersectional analyses that were precluded in smaller-scale studies would be enabled by such large-scale data collection efforts, illuminating disparities experienced by racial/ ethnic minority SGM populations. Cities should prioritize sexual orientation and gender identity data collection, regarding not only morbidity and mortality but also social and material needs [62].

Act Now and Plan for Later As supported by the reported shortages and needs within our sample, certain emergency measures can be taken immediately to reduce COVID-19 morbidity and mortality among marginalized populations. These include provision of food and emergency supplies, expansion of medical services in underserved areas, and more widespread access to PPE. However, given that these disparities are caused by structural racism and other inequities, long-term 
measures must be taken to reduce overall systemic inequality to ensure preparation for future pandemics. That is, given that pandemics can magnify existing health disparities [10], it will be most effective to take the actions to reduce these disparities overall through large structural change.

Given the evident disparities in treatment access, disease exposure, and impact, equity for racial/ethnic, gender, and sexual minority populations must be furthermore centered in official pandemic preparedness plans using an explicitly anti-racist, health equityfocused lens to prevent these disparities from occurring in future outbreaks. Using guidance from critical stakeholders including community organizations and leaders, disaster response experts, and healthcare providers, we recommend provisions be added to current planning documents to ensure equity in future pandemics and disasters and to mitigate harms that may be disproportionately felt by certain groups [33].

\section{Focus on Structural-Level Change to Support Individu-} al and Community Level Prevention Our study showed disparities in preventive behaviors by race/ethnicity. Due to the quantitative methodology of our study, we are unable to uncover the reasons for the lower levels of adherence to certain behaviors among these populations. However, it is likely that structural barriers, rather than individual choice, are driving this disparity. As such, instead of simply emphasizing individual-level prevention behaviors, such as hand washing and physical distancing, governmental bodies should implement structural change to aid in the promotion of positive health behaviors, including the ability to take off work if one is experiencing COVID-19 symptoms, expanded medical services including telehealth, and being able to consistently and accurately use PPE. Addressing these overarching structural barriers is critical to ensuring an equitable pandemic response.

\section{Conclusion}

Given the ongoing disparities experienced by marginalized populations, within and outside of the context of the COVID-19 pandemic, it is critical to take actions to ensure that the needs of these groups are met. Our results demonstrate a number of pressing unmet needs, especially for Black, Latinx, and gender minority communities within an urban setting. However, with both immediate action and consistent, structural-level investment in dismantling oppressive systems, these inequities can be addressed, not only to ensure an appropriate COVID-19 response but also to ensure longterm health equity for marginalized populations.

Supplementary Information The online version contains supplementary material available at https://doi.org/10.1007/s11524020-00497-9.

Acknowledgements The authors wish to thank the City of Chicago for their role in creating and administering the COVID19 Resiliency survey.

Funding This project received no funding.

\section{References}

1. Alegria M, Chatterii P, Wells K, Cao Z, Chen CN, Takeuchi $\mathrm{D}$, et al. Disparity in depression treatment among racial and ethnic minority populations in the United States. Psychiatr Serv. 2008;59(11):1264-72. https://doi.org/10.1176/appi. ps.59.11.1264.

2. Aranda F, Matthews AK, Hughes TL, Muramatsu N, Wilsnack SC, Johnson TP, et al. Coming out in color: racial/ethnic differences in the relationship between level of sexual identity disclosure and depression among lesbians. Cult Divers Ethn Minor Psychol. 2015;21(2):247-57. https://doi.org/10.1037/a0037644.

3. Artiga S, Garfield R, Orgera K. Communities of color at higher risk for health and economic challenges due to COVID-19. San Francisco, CA; 2020 Retrieved from https://www.kff.org/coronavirus-covid-19/issuebrief/communities-of-color-at-higher-risk-for-health-andeconomic-challenges-due-to-covid-19/. Accessed 28 June 2020.

4. Ashley, F. (2021). Trans' is my gender modality: a modest terminological proposal. In L. Erickson-Schroth (Ed.), Trans Bodies, Trans Selves. Oxford University

5. Asmundson GJG, Paluszek MM, Landry CA, Rachor GS, Mc Kay D, Taylor S. Do pre-existing anxiety-related and mood disorders differentially impact COVID-19 stress responses and coping? J Anxiety Disord. 2020;74:102271.

6. Azar KMJ, Zijun S, Romanelli R, Lockhart SH, Smits K, Robinson S, et al. Disparities in outcomes among COVID19 patients in a large health care system in California. Health Aff (Millwood). 2020; https://doi.org/10.1377 /hlthaff.2020.00598.

7. Bacak V, Thurman K, Eyer K, Qureshi R, Bird JDP, Rivera $\mathrm{LM}$, et al. Incarceration as a health determinant for sexual 
orientation and gender minority persons. Am J Public Health. 2018;108(8):994-9. https://doi.org/10.2105 /Ajph.2018.304500.

8. Beach LB, Elasy TA, Gonzales G. Prevalence of selfreported diabetes by sexual orientation: results from the 2014 behavioral risk factor surveillance system. $L G B T$ Health. 2018a;5:121-30. https://doi.org/10.1089 /lgbt.2017.0091.

9. Beach LB, Greene GJ, Lindeman P, Johnson AK, Adames $\mathrm{CN}$, Thomann M, et al. Barriers and facilitators to seeking HIV services in Chicago among young men who have sex with men: perspectives of HIV service providers. AIDS Patient Care STDs. 2018b;32(11):468-76. https://doi. org/10.1089/apc.2018.0094.

10. Blumenshine P, Reingold A, Egerter S, Mockenhaupt R, Braveman P, Marks J. Pandemic influenza planning in the United States from a health disparities perspective. Emerg Infect Dis. 2008;14(5):709-15. https://doi.org/10.3201 /eid1405.071301.

11. Bostwick WB, Boyd CJ, Hughes TL, McCabe SE. Dimensions of sexual orientation and the prevalence of mood and anxiety disorders in the United States. Am J Public Health. 2010;100(3):468-75. https://doi. org/10.2105/ajph.2008.152942.

12. Bowleg L. The problem with the phrase women and minorities: intersectionality-an important theoretical framework for public health. Am J Public Health. 2012;102(7):126773. https://doi.org/10.2105/AJPH.2012.300750.

13. Bowleg L. We're not all in this together: on COVID-19, intersectionality, and structural inequality. Am J Public Health. 2020;110(7):917. https://doi.org/10.2105 /AJPH.2020.305766.

14. Call KT, McAlpine DD, Garcia CM, Shippee N, Beebe T, Adeniyi TC, et al. Barriers to care in an ethnically diverse publicly insured population: is health care reform enough? Med Care. 2014;52(8):720-7. https://doi.org/10.1097 /MLR.0000000000000172.

15. Centers for Disease Control and Prevention. Coronavirus disease 2019 (COVID-19) cases \& deaths in the US. Atlanta, GA; 2020a.

16. Centers for Disease Control and Prevention. COVID-19 in racial and ethnic minority groups. Atlanta, GA; 2020b.

17. Centers for Disease Control and Prevention. HIV and African Americans. Atlanta, GA; 2020c.

18. Conron KJ, Mimiaga MJ, Landers SJ. A population-based study of sexual orientation identity and gender differences in adult health. Am J Public Health. 2010;100(10):1953-60. https://doi.org/10.2105/AJPH.2009.174169.

19. Egede LE, Walker RJ. Structural racism, social risk factors, and Covid-19-a dangerous convergence for Black Americans. N Engl J Med. 2020;383:e77. https://doi. org/10.1056/NEJMp2023616.

20. Friedman MR, Dodge B, Schick V, Herbenick D, Hubach RD, Bowling J, et al. From bias to bisexual health disparities: attitudes toward bisexual men and women in the United States. LGBT Health. 2014;1(4):309-18. https://doi. org/10.1089/lgbt.2014.0005.

21. Ghabrial MA. "Trying to figure out where we belong": narratives of racialized sexual minorities on community, identity, discrimination, and health. Sex Res Soc Policy.
2017;14(1):42-55. https://doi.org/10.1007/s13178-0160229-x.

22. Hanna B, Desai R, Parekh T, Guirguis E, Kumar G, Sachdeva R. Psychiatric disorders in the U.S. transgender population. Ann Epidemiol. 2019;39:1-7 e1. https://doi. org/10.1016/j.annepidem.2019.09.009.

23. Haselkorn T, Lee JH, Mink DR, Weiss ST, Grp TS. Racial disparities in asthma-related health outcomes in severe or difficult-to-treat asthma. Ann Allergy Asthma Immunol. 2008;101(3):256-63. https://doi.org/10.1016/S1081-1206 (10)60490-5.

24. Hawks L, Woolhandler S, McCormick D. COVID-19 in prisons and jails in the United States. JAMA Intern Med. 2020;180:1041-2. https://doi.org/10.1001 /jamainternmed.2020.1856.

25. Horesh D, Brown AD. Traumatic stress in the age of COVID-19: a call to close critical gaps and adapt to new realities. Psychol Trauma. 2020;12(4):331-5. https://doi. org/10.1037/tra0000592.

26. Huang Y, Zhao N. Generalized anxiety disorder, depressive symptoms and sleep quality during COVID-19 outbreak in China: a web-based cross-sectional survey. Psychiatry Res. 2020;288:112954. https://doi.org/10.1016/j. psychres.2020.112954.

27. Human Rights Campaign. The economic impact of COVID19 on the LGBTQ community in city of Chicago. Washington, DC; 2020. COVID 19 Latest Data.

28. Hutchins SS, Fiscella K, Levine RS, Ompad DC, McDonald M, Prevention's CDC. Protection of racial/ethnic minority populations during an influenza pandemic. Am J Public Health. 2009;99:S261-70. https://doi.org/10.2105 /Ajph.2009.161505.

29. Kochhar R, Cilluffo A. Key findings on the rise in income inequality within America's racial and ethnic groups. 2018.

30. Laster Pirtle WN. Racial capitalism: a fundamental cause of novel coronavirus (COVID-19) pandemic inequities in the United States. Health Educ Behav. 2020; https://doi. org/10.1177/1090198120922942.

31. Lipson SK, Raifman J, Abelson S, Reisner SL. Gender minority mental health in the U.S.: results of a national survey on college campuses. Am J Prev Med. 2019;57(3): 293-301. https://doi.org/10.1016/j.amepre.2019.04.025.

32. Lopez MH, Lee R, Budiman A. Financial and health impacts of COVID-19 vary widely by race and ethnicity. 2020 .

33. Lorenc T, Oliver K. Adverse effects of public health interventions: a conceptual framework. J Epidemiol Community Health. 2014;68(3):288-90. https://doi.org/10.1136/jech2013-203118.

34. Macapagal K, Bhatia R, Greene GJ. Differences in healthcare access, use, and experiences within a community sample of racially diverse lesbian, gay, bisexual, transgender, and questioning emerging adults. LGBT Health. 2016;3(6):434 42. https://doi.org/10.1089/lgbt.2015.0124.

35. Manuel JI. Racial/ethnic and gender disparities in health care use and access. Health Serv Res. 2018;53(3):1407-29. https://doi.org/10.1111/1475-6773.12705.

36. Marrone S. Understanding barriers to health care: a review of disparities in health care services among indigenous populations. Int J Circumpolar Health. 2007;66(3):188-98. https://doi.org/10.3402/ijch.v66i3.18254. 
37. Miller IF, Becker AD, Grenfell BT, Metcalf CJE. Disease and healthcare burden of COVID-19 in the United States. Nat Med. 2020;26:1212-7. https://doi.org/10.1038/s41591020-0952-y.

38. Myers HF, Wyatt GE, Ullman JB, Loeb TB, Chin D, Prause $\mathrm{N}$, et al. Cumulative burden of lifetime adversities: trauma and mental health in low-SES African Americans and Latino/as. Psychol Trauma-Theory Res Pract Policy. 2015;7(3):243-51. https://doi.org/10.1037/a0039077.

39. Nelson A. Unequal treatment: confronting racial and ethnic disparities in health care. J Natl Med Assoc. 2002;94(8): 666-8.

40. Nicola M, Alsafi Z, Sohrabi C, Kerwan A, Al-Jabir A, Iosifidis $\mathrm{C}$, et al. The socio-economic implications of the coronavirus pandemic (COVID-19): a review. Int J Surg. 2020;78:185-93. https://doi.org/10.1016/j.ijsu.2020.04.018.

41. Nkansah-Amankra S, Agbanu SK, Miller RJ. Disparities in health, poverty, incarceration, and social justice among racial groups in the United States: a critical review of evidence of close links with neoliberalism. Int J Health Serv. 2013;43(2):217-40. https://doi.org/10.2190/HS.43.2.c.

42. Operario D, Gamarel KE, Grin BM, Lee JH, Kahler CW, Marshall BDL, et al. Sexual minority health disparities in adult men and women in the United States: National Health and Nutrition Examination Survey, 2001-2010. Am J Public Health. 2015;105(10):E27-34. https://doi.org/10.2105 /Ajph.2015.302762.

43. Orsi JM, Margellos-Anast H, Whitman S. Black-White health disparities in the United States and Chicago: a 15year progress analysis. Am J Public Health. 2010;100(2): 349-56. https://doi.org/10.2105/Ajph.2009.165407.

44. Perrin A, Tunner E. Smartphones help blacks, Hispanics bridge some-but not all-digital gaps with whites. Washington, DC; 2019.

45. Pool LR, Ning HY, Lloyd-Jones DM, Allen NB. Trends in racial/ethnic disparities in cardiovascular health among US adults from 1999-2012. J Am Heart Assoc. 2017;6(9): e006027. https://doi.org/10.1161/JAHA.117.006027.

46. Primm AB, Vasquez MJT, Mays RA, Sammons-Posey D, McKnight-Eily, LR, Presley-Cantrell LR, ... Perry GS (2010). The role of public health in addressing racial and ethnic disparities in mental health and mental illness. Prev Chronic Dis, 7(1).

47. Raifman MA, Raifman JR. Disparities in the population at risk of severe illness from COVID-19 by race/ethnicity and income. Am J Prev Med. 2020;59(1):137-9.

48. Rooks RN, Simonsick EM, Klesges LM, Newman AB, Ayonayon HN, Harris TB. Racial disparities in health care access and cardiovascular disease indicators in black and white older adults in the health $\mathrm{ABC}$ study. J Aging Health. 2008;20(6):599-614. https://doi.org/10.1177 /0898264308321023.

49. Rosenstock S, Whitman S, West JF, Balkin M. Racial disparities in diabetes mortality in the 50 most populous US cities. J Urban Health-Bull New York Acad Med.
2014;91(5):873-85. https://doi.org/10.1007/s11524-0139861-4.

50. Ryan C, Huebner D, Diaz RM, Sanchez J. Family rejection as a predictor of negative health outcomes in White and Latino lesbian, gay, and bisexual young adults. Pediatrics. 2009;123(1):346-52. https://doi.org/10.1542/peds.20073524.

51. Steele LS, Daley A, Curling D, Gibson MF, Green DC, Williams CC, et al. LGBT identity, untreated depression, and unmet need for mental health services by sexual minority women and trans-identified people. J Womens Health. 2017;26(2):116-27. https://doi.org/10.1089/jwh.2015.5677.

52. Streed CG, Grasso C, Reisner SL, Mayer KH. Sexual orientation and gender identity data collection: clinical and public health importance. Am J Public Health . 2020;110(7):991-3. https://doi.org/10.2105/Ajph.2020.305722.

53. Talley AE, Turner B, Foster AM, Phillips G 2nd. Sexual minority youth at risk of early and persistent alcohol, tobacco, and marijuana use. Arch Sex Behav. 2019;48(4):107386. https://doi.org/10.1007/s10508-018-1275-7.

54. The Williams Institute. LGBT poverty in the United States. Retrieved from Los Angeles, CA; 2019.

55. Turner-Musa J, Ajayi O, Kemp L. Examining social determinants of health, stigma, and COVID-19 disparities. Healthcare (Basel). 2020;8(2):168. https://doi.org/10.3390 /healthcare8020168.

56. U.S. Bureau of Labor Statistics. Labor force characteristics by race and ethnicity, 2018. Washington, DC; 2019. Retrieved from https://www.bls.gov/opub/reports/race-andethnicity $/ 2018 /$ home.htm \#: : text $=$ The $\% 20$ employment $\%$ E2\% $80 \% 93$ population $\% 20$ ratio $\% 20$ was, and $\% 2063.2 \% 20$ percent $\% 20$ for $\% 20 \mathrm{Hispanics}$. Accessed 28 June 2020.

57. United States Census Bureau. QuickFacts Chicago, Illinois. Suitland-Silver Hill, MD; 2019.

58. van Dorn A, Cooney RE, Sabin ML. COVID-19 exacerbating inequalities in the US. Lancet. 2020;395(10232):12434.

59. Vega WA, Rodriguez MA, Gruskin E. Health disparities in the Latino population. Epidemiol Rev. 2009;31(1):99-112. https://doi.org/10.1093/epirev/mxp008.

60. Yancy CW. COVID-19 and African Americans. Jama-J Am Med Assoc. 2020;323(19):1891-2. https://doi.org/10.1001 /jama.2020.6548.

61. City of Chicago. COVID-19 Latest Data. 2020.

62. Phillips II G, Felt D, Ruprecht MM, Wang X, Xu J, PérezBill E, Bagnarol RM, Roth J, Curry CW, Beach LB. Addressing the Disproportionate Impacts of the COVID-19 Pandemic on Sexual and Gender Minority Populations in the United States: Actions Toward Equity. LGBT Health. 2020;7:279-82.

Publisher's Note Springer Nature remains neutral with regard to jurisdictional claims in published maps and institutional affiliations. 